\title{
Lymph Node Radiotherapy Instead of Extended Axillary Surgery - the New Standard?
}

\author{
Peter Niehoff Silla Hey-Koch \\ Department of Radiation Oncology, Sana Klinikum Offenbach, Offenbach, Germany
}

\begin{abstract}
Keywords
Adjuvant treatment · Breast cancer - Lymph nodes . Radiotherapy

\section{Summary}

Breast cancer treatment has undergone major changes in the last 20 years. Specifically, the role of axillary lymph node dissection has changed from radical axillary dissection with excision of a high number of lymph nodes to sentinel lymph node biopsy (SLNB). This paradigm shift is associated with a controversial debate regarding the significance of axillary staging, the need for surgery, and the role of radiotherapy. Looking ahead, lymph node staging and axillary treatment might shift from SLNB and/or axillary dissection to ultrasoundguided needle biopsy and irradiation of regional lymph nodes in order to reduce treatment-related sequelae in early-stage breast cancer.
\end{abstract}

(C) 2018 S. Karger GmbH, Freiburg

\section{Decreasing Axillary Staging}

In previous years, the number of positive lymph nodes was an important criterion to indicate systemic therapy [1]. However, recently, biological markers and tumor biology have been rapidly gaining importance [2].

Halsted's hypothesis of radical mastectomy included the removal of the breast, the major and minor pectoralis muscle, as well as the ipsilateral axillary lymph nodes [3]. However, despite being lymph node-negative, $30 \%$ of patients developed locoregional relapse and even distant metastases [4].

\section{KARGER}

(c) 2018 S. Karger GmbH, Freiburg
The NSABP B-04 trial randomized 1,079 patients to radical mastectomy with axillary radiotherapy (ART), mastectomy with axillary dissection (AD), or mastectomy alone with observation of the axilla. The 10 -year results showed a $17.8 \%$ axillary recurrence rate in patients with untreated axillae compared to $1.4 \%$ in those who underwent $\mathrm{AD}$ and $3.1 \%$ in those with $\mathrm{ART}$ [5]. Still, AD remained the treatment of choice for axillary management, mainly because the number of involved nodes guided the decision regarding adjuvant systemic therapy.

However, due to improved screening, the prevalence of axillary metastases has decreased. Considering the high risk of surgical morbidity, with a lymphedema incidence of up to $50 \%$ after 20 years, and the increasing importance of biological markers, extensive surgical treatment became more and more questionable [6-11].

Sentinel lymph node (SLN) biopsy (SLNB) represents an opportunity to reduce axillary morbidity while obtaining lymph node staging. Several trials have proven the safety of omitting AD in the case of a negative SLNB. In a systematic review published in 2010 with over 15,000 patients, the axillary recurrence rate was $0-3 \%$ at a mean follow-up of 34 months [12].

In the NSABP B-32 trial with more than 5,600 patients with early breast cancer, more than 3,900 patients were SLN-negative and did not undergo further axillary treatment. The remaining SLN-positive patients were randomized to 2 groups: AD versus observation. Among the 3 groups, no differences in overall survival (OS), disease-free survival (DFS), and locoregional control could be found [13].

With the introduction of SLNB, the rate of lymph node positivity went up to $30 \%$. The more detailed pathology workup $[14,15]$ identified a higher number of small tumor foci. However, initially, the clinical impact of these small foci remained unclear [15]. Therefore, the IBCSG 23-01 and the AATRM trial randomized patients with SLN micrometastases to either further axillary surgery or observation. In both studies, regional failure and OS were statistically equivalent between groups $[16,17]$. 


\section{Axillary Radiotherapy: An Alternative Option for the Adjuvant Axillary Management of Breast Cancer}

For a long time, the usage of regional lymph node irradiation in early breast cancer was very restrictive. Most international guidelines advised radiotherapy of the lymphatics only in the case of more than 3 positive lymph nodes. The commonly recommended treatment volume merely encompassed the supraclavicular fossa. This cautious approach was mainly based on insufficient data regarding the benefit of additional lymph node irradiation in terms of survival and the supposed risk of side effects such as lymphedema and plexopathy. A meta-analysis demonstrated an average incidence of treatment-related lymphedema in up to $20 \%$ of patients with early breast cancer [18]. The pooled data identified the extent of axillary surgery as the leading risk factor for lymphedema. These findings were confirmed in a large population-based cohort of nearly 1,800 breast cancer patients [19]. The authors found no lymphedema events within the group of patients without any axillary surgery. However, the 5 -year incidence of lymphedema increased with the degree of surgery from $5.3 \%$ in the case of SLNB to $15.9 \%$ in the case of AD (p $<0.001$ ). In multivariate analysis, radiotherapy, chemotherapy, and obesity were identified as additional factors further increasing the risk of lymphedema in conjunction with any axillary surgery.

Therefore, complete omission of $\mathrm{AD}$ in the case of positive SLNB has been the next progressive step in order to reduce treatment-related side effects. The ACOSOG-Z0011 and the AMAROS trial randomized patients with limited numbers of positive SLN $[20,21]$ comparing AD to no further therapy or non-invasive treatment. The ACOSOG-Z0011 trial recruited 891 patients treated with breast-conserving therapy followed by adjuvant systemic treatment and tangential radiotherapy. Irradiation of the lymphatics was not permitted per protocol. Regarding OS and regional control, AD showed no additional benefit. The AMAROS trial compared AD versus ART in over 1,425 SLN-positive patients after breast-conserving surgery or mastectomy. Likewise, this trial reported no additional benefit of surgery compared to non-invasive treatment. However, the surgical approach led to an almost 2-fold increase in lymphedema compared to radiotherapy.

The randomized, single-center OTOASOR study compared AD and ART and yielded similar results in <CT3 SLNB-positive patients. After 8 years of follow-up, there was no statistical difference in axillary recurrence or DFS between the 2 treatment arms [22].

However, is radiotherapy at all beneficial in patients with lowvolume lymph node metastases? In fact, a meta-analysis by Budach et al. [23] confirmed this in 2015. The authors pooled the data of 3 randomized trials: MA.20, EORTC-22922/10925, and a French trial [24-26]. The major eligibility criterion of all 3 trials was $\mathrm{pN}+$. The MA.20 also included lymph node-negative patients with a high risk for recurrence, while the EORTC and the French trial included lymph node-negative patients with a medial or central tumor location. The primary endpoint was OS, secondary endpoints were DFS and distant metastasis-free survival (DMFS). MA.20 and EORTC-22922/10925 both showed an improvement in DFS and, interestingly, DMFS, but no significant impact on OS. However, the meta-analysis of Budach et al. [23] showed that additional radiotherapy to the internal mammary and/or supraclavicular lymph nodes resulted in significantly improved OS and DFS; however, the absolute benefit was low.

The influence of the extent of nodal irradiation was also demonstrated by the DBCG-IMN study [27] which was performed as a population-based approach with radiotherapy to the internal mammary chain in addition to the supraclavicular fossa for rightsided breast cancer only. The results demonstrated improved OS for the extended treatment volume. However, the significant improvement in the 8-year survival rate was only found in premenopausal patients. Tumor biology seems to influence the importance of the extent of nodal irradiation.

\section{Discussion}

The increasing number of patients diagnosed with early breast cancer and their excellent prognosis have resulted in less invasive treatments in order to decrease late sequelae. The evidence for omission of $\mathrm{AD}$ without impairment of local control and OS is still weak. The ACOSOG Z0011 trial seemed to prove its safety as presented by the authors in the 10-year update [28]. However, the study had several limitations. The trial was underpowered to reach statistical significance concerning its primary endpoint, OS, and the dropout rate almost reached $20 \%$ [29]. In the $\mathrm{AD}$ arm, micrometastases $(<2 \mathrm{~mm})$ were identified in only $37.5 \%$ of the patients compared to $44.8 \%$ in the SLNB group $(\mathrm{p}=0.046)$. Furthermore, an analysis of the 228 available radiotherapy plans revealed a substantial amount of protocol violations. In the SLNB-only arm, more than $18 \%$ of the patients received prohibited irradiation of the axilla or the supraclavicular fossa, and almost $50 \%$ of the patients were treated with 'high tangential fields' increasing the dose to the axillary level II.

The American Society of Oncology, the St. Gallen Consensus, and the recently updated Germany Cancer Society guidelines advise to omit $\mathrm{AD}$ in the case of 1-2 positive lymph nodes. For patients with more than 2 positive lymph nodes, radiotherapy is considered as an alternative to surgery.

The authors of a systematic review and meta-analysis concluded that SLNB only is an adequate treatment for patients with up to 2 micrometastases. For those patients with macrometastases in the SLNB, omission of AD is a feasible option provided that the patients receive adequate adjuvant treatment including adjuvant radiotherapy to the breast [30].

A further step towards reducing late sequelae could be the omission of surgical intervention in the clinically negative axilla altogether.

The ongoing German INSEMA trial is investigating a restrictive axillary staging in clinically node-negative early breast cancer patients [31].

The results of the SOUND trial might lead to the replacement of any open surgical staging by ultrasound-guided core or needle biopsy of negative or sonographically suspicious lymph nodes for early breast cancer smaller than $2 \mathrm{~cm} \mathrm{[32].}$ 
The ongoing POSNOC trial may finally answer the question whether no further axillary treatment, $\mathrm{AD}$, or ART should be the treatment of choice for SLN-positive breast cancer patients [33]. However, this study is not expected to complete accrual before 2018.

\section{Conclusion}

Considering i) the evidence proving the importance of nodal irradiation, ii) the low rates of toxicity compared to extensive surgery, and iii) the absence of randomized trial results demonstrating in which cases additional axillary treatment can be safely omitted, we believe that ART is a safe and less toxic alternative to AD.

The role of radiotherapy to the axilla in the setting of complete clinical response of initially positive axillary lymph nodes after ne- oadjuvant radiotherapy remains less clear. The ALLIANCE A011202 trial (ClinicalTrials.gov: NCT01901094) might clarify this role. The trial is still recruiting patients presenting with $\mathrm{cT} 1-3 \mathrm{cN} 1$ and clinical complete remission after neoadjuvant chemotherapy. These patients undergo SLNB before randomization to axillary clearance or radiotherapy. Ongoing trials such as SERC/IPC 2012001 (ClinicalTrials.gov: NCT01717131) in France, SENOMAC (ClinicalTrials.gov: NCT02240472) in Sweden, and BOOG 2013-07 (ClinicalTrials.gov: NCT 0211682) in the Netherlands currently investigate the same topic of omitting axillary surgery. The results of these trials are expected in 2027-2029.

\section{Disclosure Statement}

The authors do not have any conflicts of interest to declare.

\section{References}

1 Carter CL, Allen C, Henderson DE: Relation of tumour size, lymph node status and survival in 24,740 breast cancer cases. Cancer 1989;73:505-508.

2 Caldos C, Aparicio SA: The molecular outlook. Nature 2002;415:484-485

3 Halsted CP, Benson JR, Jatoi I: A historical account of breast cancer surgery: beware of local recurrence but be not radical. Fut Oncol 2014;10:1649-1657.

4 Halsted WS: The results of operation for the cure of cancer of the breast performed at the John Hopkins Hospital from June 1889 to January 1894. Ann Surg 1894;20:497-555.

5 Fisher B, Montague F, Redmond C, et al: Ten-year results of a randomized trial comparing radical mastectomy and total mastectomy with or without radiation. N Engl J Med 1985;312:674-681.

6 Early Breast Cancer Trialists' Collaborative Group (EBCTCG): Effects of chemotherapy and hormonal therapy for early breast cancer on recurrence and 15year survival: an overview of the randomised trials. Lancet 2005;365:1687-1717.

7 Hofvind S, Lee CI, Elmore JG: Stage-specific breast cancer incidence rates among participants and non-participants of a population-based mammographic screening program. Breast Cancer Res Treat 2012;135:291-299.

8 Ivens D, Hoe AL, Podd TJ, et al: Assessment of morbidity from complete axillary dissection. Br J Cancer 1992;66:136-138.

$\checkmark$ Pain SJ, Purushotham AD: Lymphoedema following surgery for breast cancer. Br J Surg 2000;87:1128-1141.

10 Petrek JA, Senie RT, Peters M, et al: Lymphedema in a cohort of breast carcinoma survivors 20 years after diagnosis. Cancer 2001;92:1368-1377.

11 Purushotham AD, MacMillan RD, Wishart G: Advances in axillary surgery for breast cancer - time for a tailored approach. Eur J Surg Oncol 2005;31:929-931.

12 Van der Ploeg IMC, Nieweg OE, van Rijk MC, et al: Axillary recurrence after a tumour negative sentinel lymph node biopsy in breast cancer patients: a systematic review and meta-analysis of the literature. Eur J Surg Oncol 2008;34:1277-1284.

13 Krag D, Anderson SJ, Julian TB, et al: Sentinel lymph node resection compared with conventional axillary lymph node dissection in clinically node negative patients with breast cancer: overall survival findings from the NSABP B-32 randomised phase 3 trial. Lancet Oncol 2010;11:927-933.
14 Cserni G: Complete sectioning of axillary sentinel nodes in patients with breast cancer with breast cancer. Analysis of two different step-sectioning and immunohistochemistry protocols in 246 patients. J Clin Pathol 2002;55:926-931.

15 Tvedskov TF: Staging of women with breast cancer after introduction of sentinel node guided axillary dissection. Dan Med J 2012;59:B4475.

16 Galimberti V, Cole BF, Zurrida S, et al: Update of International Breast Cancer Study Group Trial 23-01 to compare axillary dissection versus no axillary dissection in patients with clinically node negative breast cancer and micrometastases in the sentinel node. Cancer Res 2011;71:102s.

17 Sola M, Alberro JA, Fraile M, et al: Complete axillary lymph node dissection versus clinical follow up in breast cancer patients with sentinel node metastasis: final results from the multicentre clinical trial AATRM 048/13/2000. Ann Surg Oncol 2013;20:120-127.

18 DiSipio T, Rye S, Newman B, Hayes S: Incidence of unilateral arm lymphoedema after breast cancer: a systematic review and meta-analysis. Lancet Oncol 2013; 14:500-515.

19 Nguyen T, Hoskin TL, Habermann EB, et al: Breast cancer-related lymphedema risk is related to multidisciplinary treatment and not surgery alone: results from a large cohort study. Ann Surg Oncol 2017;24:2972-2980.

20 Giuliano AE, Hunt K, Ballman K, et al: Axillary dissection vs no axillary dissection in women with invasive breast cancer and sentinel node metastases: a randomized clinical trial. JAMA 2011;305:569.

21 Donker M, van Tienhoven G, Straver ME, et al: Radiotherapy or surgery of the axilla after a positive sentinel node in breast cancer (EORTC 10981-22023 AMAROS): a randomized multicentre, open-label, phase 3 non-inferiority trial. Lancet Oncol 2014;15:1303-1310.

22 Sávolt Á, Péley G, Polgár C, et al: Eight-year follow up result of the OTOASOR trial: the Optimal Treatment Of the Axilla - Surgery Or Radiotherapy after positive sentinel lymph node biopsy in early-stage breast cancer. Eur J Surg Oncol 2017;43:672-679.

23 Budach W, Bölke E, Kammers K, et al: Adjuvant radiation therapy of regional lymph nodes in breast cancer - a meta-analysis of randomized trials - an update. Radiat Oncol 2015;10:258.
24 Whelan TJ, Olivotto IA, Parulekar WR, et al.; MA.20 Study Investigators: Regional nodal irradiation in earlystage breast cancer. N Engl J Med 2015;373:307-316.

25 Poortmans PM, Collette S, Kirkove C, et al.; EORTC Radiation Oncology and Breast Cancer Groups: Internal mammary and medial supraclavicular irradiation in breast cancer. N Engl J Med 2015;373:317-327.

26 Hennequin C, Bossard N, Servagi-Vernat S, et al: Ten year survival results of a randomized trial of irradiation of internal mammary nodes after mastectomy. Int J Radiat Oncol Biol Phys 2013;86:860-866.

27 Thorsen LB, Offersen BV, Danø H, et al: DBCG-IMN a population-based cohort study on the effect of internal mammary node irradiation in early node-positive breast cancer. J Clin Oncol 2016;34:314-320.

28 Giuliano AE, Ballman KV, McCall L, et al: Effect of axillary dissection vs no axillary dissection on 10-year overall survival among women with invasive breast cancer and sentinel node metastasis: the ACOSOG Z0011 (Alliance) randomized clinical trial. JAMA 2017;318:918-926.

29 Glechner A, Wöckel A, Gartlehner G, et al: Sentinel lymph node dissection only versus complete axillary lymph node dissection in early invasive breast cancer: a systematic review and meta-analysis. Eur J Cancer 2013;49:812-825.

30 Ram R, Singh J, McCaig E: Sentinel node biopsy alone versus completion axillary node dissection in node positive breast cancer: systematic review and metaanalysis. Int J Breast Cancer 2014;2014:513780.

-31 Reimer T, Stachs A, Nekljudova V, et al: Restricted axillary staging in clinically and sonographically nodenegative early invasive breast cancer (c/iT1-2) in the context of breast conserving therapy: first results following commencement of the Intergroup-SentinelMamma (INSEMA) trial. Geburtshilfe Frauenheilkd 2017;77:149-157.

32 Jones P, Jones S, Mills P, et al: Identification of sentinel lymph nodes preoperatively using microbubble enhanced ultrasound in patients with breast cancer. Proc Am Soc Clin Oncol 2009; abstr 44

33 Goyal A, Dodwell D: POSNOC: a randomised trial looking at axillary treatment in women with one or two sentinel nodes with macrometastases. Clin Oncol (R Coll Radiol) 2015;27:692-695. 